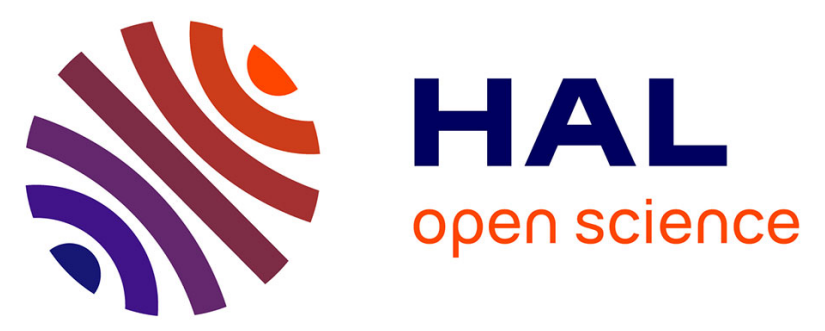

\title{
Mineral- and Ion-Specific Effects at Clay-Water Interfaces: Structure, Diffusion, and Hydrodynamics
}

Pauline Simonnin, Virginie Marry, Benoit Noetinger, Carlos Nieto-Draghi, Benjamin Rotenberg

\section{- To cite this version:}

Pauline Simonnin, Virginie Marry, Benoit Noetinger, Carlos Nieto-Draghi, Benjamin Rotenberg. Mineral- and Ion-Specific Effects at Clay-Water Interfaces: Structure, Diffusion, and Hydrodynamics. Journal of Physical Chemistry C, 2018, 122 (32), pp.18484 - 18492. 10.1021/acs.jpcc.8b04259 . hal-01872554

\section{HAL Id: hal-01872554 https://hal.sorbonne-universite.fr/hal-01872554}

Submitted on 12 Sep 2018

HAL is a multi-disciplinary open access archive for the deposit and dissemination of scientific research documents, whether they are published or not. The documents may come from teaching and research institutions in France or abroad, or from public or private research centers.
L'archive ouverte pluridisciplinaire HAL, est destinée au dépôt et à la diffusion de documents scientifiques de niveau recherche, publiés ou non, émanant des établissements d'enseignement et de recherche français ou étrangers, des laboratoires publics ou privés. 


\section{Mineral- and Ion-Specific Effects at Clay-Water}

\section{Interfaces: Structure, Diffusion and Hydrodynamics}

Pauline Simonnin, ${ }^{\dagger, \ddagger}$ Virginie Marry, ${ }^{\dagger}$ Benoit Noetinger,,+ Carlos Nieto-Draghi,, and Benjamin Rotenberg*,

Sorbonne Université, CNRS, Physicochimie des électrolytes et nanosystèmes interfaciaux, UMR PHENIX, F-75005, Paris, France, and IFP Energies Nouvelles, 1864 avenue de Bois-Préau, 92852 Rueil-Malmaison

E-mail: benjamin.rotenberg@sorbonne-universite.fr

\footnotetext{
${ }^{*}$ To whom correspondence should be addressed

†Sorbonne Université, CNRS, Physicochimie des électrolytes et nanosystèmes interfaciaux, UMR PHENIX, F-75005, Paris, France

$\ddagger$ IFP Energies Nouvelles, 1 \& 4 avenue de Bois-Préau, 92852 Rueil-Malmaison
} 


\begin{abstract}
We use molecular dynamics to investigate how the structure, diffusion and hydrodynamic properties of clay interfaces with aqueous solution depend on the nature of the clay, the nature of the counterions and the salt concentration in the solution. Specifically, we study water-filled nanopores between uncharged (pyrophyllite) and charged (montmorillonite and beidellite, with susbtitutions located in the octahedral and tetrahedral layers, respectively) clays, with sodium or cesium as counterions, in the absence and in the presence of added salt. We discuss how the balance between solvation and attraction of the cations to the surface results in various distributions between innerand outer-sphere complexes and how this influences the dynamics of water near the surface, as well as the hydrodynamic flow in the presence of an external force. In the latter case, the discussion based on mapping the molecular velocity profiles to a continuous description (parabolic Poiseuille flow) shows that the larger effects come from the presence/absence of charge in the mineral, as well as the localization of substitutions within the clay layer. The salt concentration and the nature of the counterions have a comparatively less important impact far from the surface - even though some differences are observed in its close vicinity, which are not properly captured by the continuous description.
\end{abstract}




\section{Introduction}

The transport of fluids through nanopores plays a role in a many environmental, biological and technological processes, ranging from clean water production or the harvesting of blue energy using membranes ${ }^{1}$ to oil and natural gas recovery, ${ }^{2}$ carbon sequestration ${ }^{3}$ or the geological disposal of radioactive waste. ${ }^{4}$ Several fundamental questions arise for the understanding and prediction of the transport properties in these contexts, such as the validity of continuum hydrodynamic theories when the confining length scale becomes comparable to the molecular size, as well as the dominant role of interfacial effects.

Molecular simulations played a key role in addressing the first aspect with the finding that continuous hydrodynamics holds down to remarkably small length scales, ${ }^{5}$ even though it breaks down beyond 1-2 $\mathrm{nm}$ in particular due to the layering of the fluid at the interface, or for pore sizes of the order of that of the confined fluid molecules. ${ }^{6}$ They also provide molecular scale information on the local viscosity of the confined fluid ${ }^{7-10}$ as well as the relevant boundary conditions (stick or slip) which should be applied for a given interface, depending on the nature of both the solid wall and the confined fluid, with the quantification of the slip length ${ }^{11-13}$ and its correlation with other properties such as the contact angle. ${ }^{14}$

Due to their importance in the above-mentioned geological applications, the dynamics of fluids in nanopores of clay and related natural minerals ${ }^{15-20}$ have attracted a lot of attention from the experimental and modelling point of view. We will not discuss here the interlayer pores, where only 1 or 2 fluid layers are present (see e.g. Refs. 21-26 for recent molecular simulation studies), but rather focus on larger pores (a few nanometers), where pressuredriven flow may provide a contribution in addition to mere diffusion. Molecular dynamics have already provided detailed information on the structure and dynamics of the interface between such charged layered aluminosilicates and aqueous solutions. Previous studies focussed e.g. on the structure and energetics of ion sorption onto external surfaces, ${ }^{27,28}$ on the relevance of continuous double- and triple-layer models to describe the ion distribution, ${ }^{29}$ on the structure and energetics of ions in the Stern layer ${ }^{30}$ or on the diffusion properties on 
the molecular (to investigate the solvent and ion dynamics within the first adsorbed layers of fluids ${ }^{31-35}$ ) and larger scales (from the local interlayer and interparticle dynamics to that through a porous medium consisting of clay particles $\left.{ }^{36}\right)$.

Using non-equilibrium molecular dynamics simulations, we have also investigated pressuredriven flow in sodium montorillonite clay nanopores. ${ }^{37}$ Due to the negative charge of the surface and the presence of counterions, electro-osmotic flows can also be induced by applied electric field, and the corresponding transport properties have also been investigated with molecular dynamics simulations. ${ }^{38-42}$ It was found in particular that the slip length measured for both types of flow are similar. Despite the small value (smaller than the size of a water molecule), neglecting such slip may result in sizeable errors in the prediction of the overall flow in nanopores.

Clay minerals further provide an ideal situation to investigate the influence of the nature of the surface and of the fluid on the hydrodynamic properties of experimentally relevant interfaces. Indeed, they can differ by the presence or absence (and density) of substitutions in the mineral framework, leading to various charge densities, as well as by the localization of these substitutions within the framework (closer to or further from the surface in contact with the fluid). Finally, the nature of the counterion compensating the charge of the mineral may also be varied, resulting in ion-specific properties. Following previous work e.g. on ion sorption ${ }^{28}$ or clay swelling, ${ }^{43}$ here we investigate the effect of the nature of the clay surface, that of the counterion and the presence of salt on the properties of clay mineral - aqueous interfaces. We focus in particular on hydrodynamic properties and on the possibility to describe the flow in the presence of an external perturbation with continuum hydrodynamics, using appropriate boundary conditions derived from molecular simulations. This requires understanding first the structure of the interfacial fluid. Systems and methods are presented in the next section. We then present equilibrium density and water diffusion profiles for the various systems, and finally discuss the hydrodynamic properties. 


\section{Methods}

\subsection{Systems}

We consider three different clay minerals: pyrophyllite, montmorillonite and beidellite. All three belong to the family of 2:1 phyllosilicates (layered aluminosilicates), where an octahedral aluminum oxide sheet is sandwiched between two tetrahedral silicon oxide sheets (see Figure 1). While pyrophillite with unit formula $\mathrm{Al}_{4} \mathrm{Si}_{8} \mathrm{O}_{20}(\mathrm{OH})_{4}$ is electrically neutral, montmorillonite and beidellite are negatively charged due to the presence of substitutions in the octahedral and tetrahedral sheets, respectively. This charge is then compensated by counterions in the interlayer. Specifically, we simulate montmorillonite and beidellite clays with identical charge densities with unit formula $\mathrm{X}_{0.75}\left(\mathrm{Al}_{3.25} \mathrm{Mg}_{0.75}\right) \mathrm{Si}_{8} \mathrm{O}_{20}(\mathrm{OH})_{4}$ and $\mathrm{X}_{0.75} \mathrm{Al}_{4}\left(\mathrm{Si}_{7.25} \mathrm{Al}_{0.75}\right) \mathrm{O}_{20}(\mathrm{OH})_{4}$, respectively, with $\mathrm{X}=\mathrm{Na}^{+}$or $\mathrm{Cs}^{+}$the compensating counterion. This allows us to investigate the combined effects of the clay charge (or absence thereof), its localization within the mineral layer and the nature of the counterion.

\subsection{Computational Details}

For each system, the simulation box (see Figure 1) contains one clay layer of 4x8 unit cells. The layer is divided in two halves and periodic boundary conditions in all directions are used. A single layer may seem insufficient to describe the interface between a clay particle (with several layers and interlayers) and a solution. Indeed, it is known that for low interlayer content the properties of bulk clay particles may depend on the number of layers used, even though the influence is already small in the bihydrated states (two water layers). ${ }^{44}$ We have checked for one of the systems that no differences were observed when considering: (a) a single clay layer, without interlayer (b) two layers and an interlayer in the bihydrated state and (c) three layers and two interlayers in the bihydrated state. This suggests that the first setup, which was then chosen for its lower computational cost, is sufficient to mimic the interface between a fluid and a clay particle in the bihydrated state. Such a state if of course relevant 


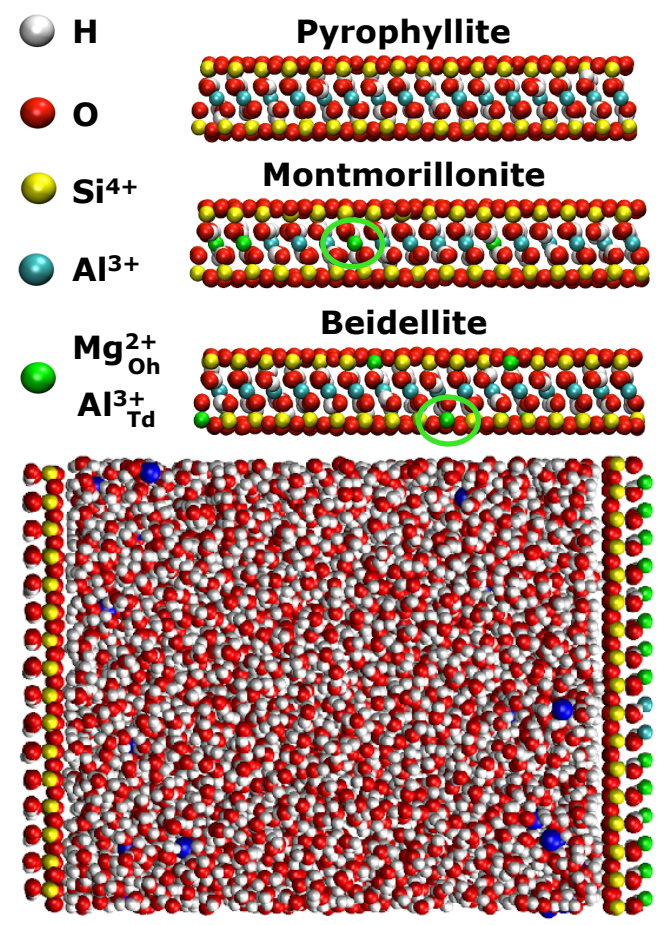

Figure 1: Top Pyrophyllite, montmorillonite and beidellite clay layers differ by the presence or absence of substitutions in the octahedral $\left(\mathrm{Al}^{3+} \rightarrow \mathrm{Mg}^{2+}\right.$, montmorillonite) and tetrahedral $\left(\mathrm{Al}^{3+} \rightarrow \mathrm{Mg}^{2+}\right.$, beidellite) sheets. Bottom Simulation box (see text for details). 
in real materials only for swelling clays such as montmorillonite and beidellite. Pyrophyllite doesn't swell in practice, but we keep the same setup for a consistent comparison between the three clays.

The negative charge of the mineral is compensated by $24 \mathrm{Na}^{+}$or $\mathrm{Cs}^{+}$counterions. In the following, we will refer to the simulated systems as $0 \mathrm{M}$ (resp. $1 \mathrm{M}$ ) if they contain no additional ions (resp. $40 \mathrm{NaCl}$ or $\mathrm{CsCl}$ ion pairs). The box size is $41.44 \times 35.88 \times 58.4 \AA^{3}$ for all systems, with a common length in the direction perpendicular to the surfaces determined from a prior 500 ps simulation for Na-montmorillonite in the $N P T$ ensemble with $P=1$ bar and $T=298 \mathrm{~K}$ using Nosé-Hoover barostat and thermostat with time constants of $2 \mathrm{ps}$ and 1 ps, respectively. The salt-free systems all contain 2436 water molecules, while their number for the salt-containing systems was adjusted to recover a concentration of $1 \mathrm{M}$ far from the surface (2408 and 2332 molecules with $\mathrm{NaCl}$ and $\mathrm{CsCl}$, respectively). This choice of imposing the bulk density in the central region, rather than the water and ions chemical potentials, is motivated by the objective of a simple hydrodynamic description of a fluid characterized by its bulk properties (most importantly for hydrodynamics, its density and viscosity), together with an interface-specific boundary condition, we have preferred to impose. We note that this results in slightly different pressures for a given pore size (to facilitate the comparison between systems).

Interactions between atoms are described by a classical, non-polarizable force field including Coulomb and Lennard-Jones (LJ) interactions. The partial charges and LJ parameters are taken from the CLAYFF force field for the clay atoms, ${ }^{15}$ from the SPC/E water model ${ }^{45}$ and from Ref 46 for the ions, together with the Lorentz-Berthelot mixing rules. ${ }^{47}$ The water molecules are treated as rigid via the SHAKE algorithm. ${ }^{48}$ Long-range electrostatic interactions are computed using the Particle-Particle-Particle-Mesh method, ${ }^{49}$ while a cut-off radius of $12 \AA$ is used for the computation of LJ interactions. Molecular dynamics simulations are performed using a time step of $1 \mathrm{fs}$. A Nose-Hoover ${ }^{50}$ thermostat with a time constant of 1 ps is applied on the clay atoms only. Energy exchanges with the fluid are then sufficient 
to thermalize the latter for both equilibrium and non-equilibrium simulations.

For each simulation box, the system is first equilibrated for $1 \mathrm{~ns}$. The density profiles are then computed from production runs of $5 \mathrm{~ns}$. The final configuration is then used as initial configuration for two sets of simulations. On the one hand, four independent trajectories of $4 \mathrm{~ns}$ each (following $2.5 \mathrm{~ns}$ of equilibration after generating different sets of random velocities), used to compute diffusion coefficients in various layers (see below) using the method described in Ref. 31. The reported uncertainties correspond to the standard deviation between these 4 independent trajectories. On the other hand, non-equilibrium simulations in which a force is applied to all atoms in the fluid (water oxygen and hydrogen, ions) are performed to sample the steady-state velocity profiles for $6 \mathrm{~ns}$, after a transient regime of $5 \mathrm{~ns}$. This common approach is not strictly equivalent to an applied pressure gradient, which corresponds to a uniform volumic force, but it has the advantage of simplicity as it does not require prior knowledge of the density profiles. In such non-equilibrium simulations, a single $\mathrm{Al}$ atom in each clay layer is constrained at its initial position to prevent the drift of the whole solid due to the liquid-solid friction.

\section{Results and discussion}

\subsection{Equilibrium properties}

\subsubsection{Structure of the interface}

We first investigate the structure of the various interfaces. Fig. 2 shows the density profiles of water oxygen and hydrogen for the three different clays with $\mathrm{Na}^{+}$counterion (similar results are obtained with $\mathrm{Cs}^{+}$), as well as the density profiles of the cations in the presence of $1 \mathrm{M}$ of added salt for both the $\mathrm{Na}^{+}$and $\mathrm{Cs}^{+}$cases. As reported previously on similar surfaces (see e.g. Ref. 31-33), water is organized with 2-3 well defined layers at the surface, beyond

which it behaves as a bulk-like fluid. The combined oxygen and hydrogen positions indicate 
that the orientation of water in the first adsorbed layer depends on the charge of the surface. While in pyrophyllite hydrogen bonds are formed almost exclusively among water molecules, as on a hydrophobic surface, ${ }^{51}$ a significant fraction of $\mathrm{OH}$ bonds are oriented toward the surface on beidellite and montmorillonite.
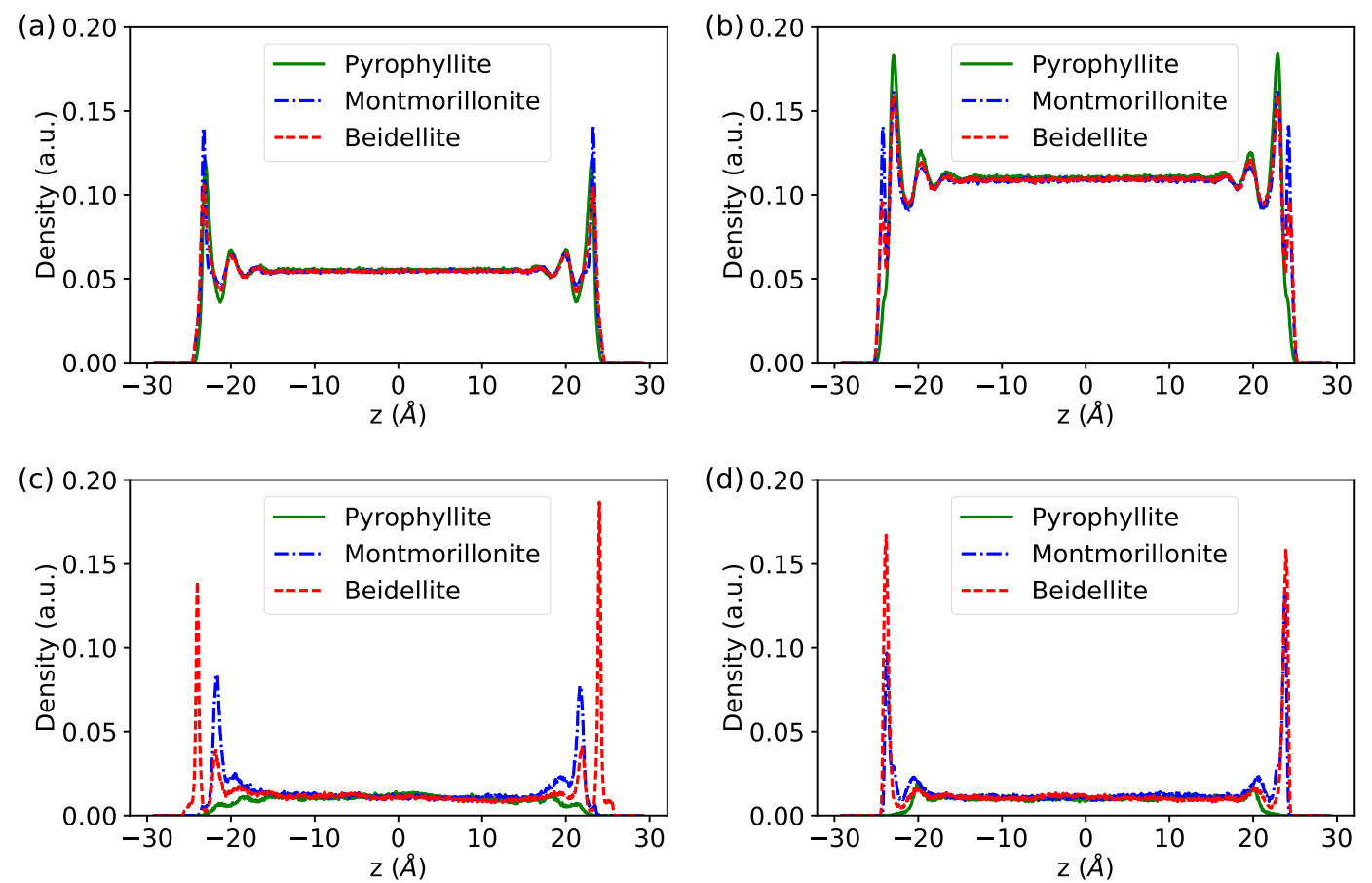

Figure 2: Distribution of (a) water oxygen, (b) water hydrogen, (c) $\mathrm{Na}^{+}$and (d) $\mathrm{Cs}^{+}$as a function of the position $z$ across the pore, for a $1 \mathrm{M}$ salt concentration and the three clays: pyrophyllite (green), montmorillonite (blue) and beidellite (red). The water densities are similar for both counterions and only the case of $\mathrm{Na}^{+}$is shown.

The ionic density profiles on negatively charged clays (montmorillonite and beidellite) indicate a depletion of $\mathrm{Cl}^{-}$(not shown) and an excess of $\mathrm{Na}^{+}$or $\mathrm{Cs}^{+}$cations at the surface, as expected. For the latter, both inner-sphere and outer-sphere complexes are observed, in proportions which vary with the localization of the charge within the mineral layer and the nature of the counterion. As one might have anticipated, ${ }^{52}$ more inner-sphere complexes, in which the ion is partly desolvated and in direct contact with surface oxygen atoms, are observed (a) when the charge is in the tetrahedral sheet (beidellite), i.e. closer to the fluid, and (b) for the larger $\mathrm{Cs}^{+}$cation, which has a smaller hydration free energy than 
$\mathrm{Na}^{+} .{ }^{53}$ These observations are consistent with the distribution of these cations in bihydrated interlayers of these clays. ${ }^{43}$ We finally note an asymmetry between both sides of the ion density profiles in the case of beidellite, which results from the asymmetry in the distribution of the substitutions within the tetrahedral sheet: Even though the number of substitutions and hence that of counterions is the same on both sides of the layer (12 each), they are more clustered on one side and more evenly distributed in the other (see Figure 3). The repulsion between adsorbed counterions then modifies their distribution in the vicinity of the attractive charge defects within the solid.

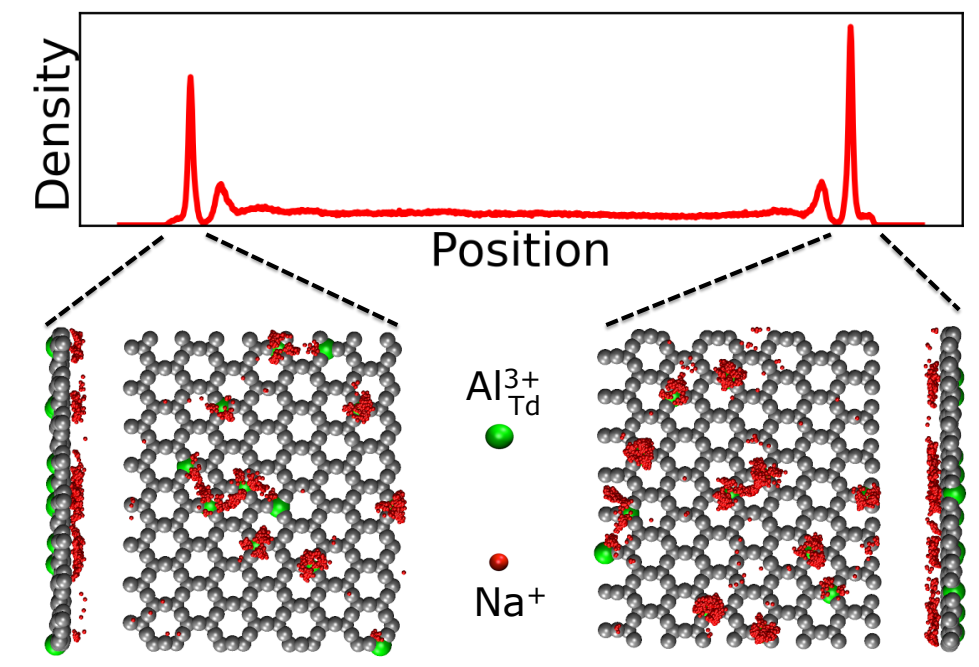

Figure 3: The asymmetric ionic density profile for beidellite (here for $\mathrm{Na}^{+}$counterions with $1 \mathrm{M} \mathrm{NaCl}$ ) arises from the asymmetry in the substitutions within the tetrahedral layer close to the surface. The bottom panels indicate the localization of the Al susbtitutions (green) within the surface $\mathrm{Si}$ and $\mathrm{O}$ atoms (grey). The positions of $\mathrm{Na}^{+}$ions (red) within the first adsorbed layers, for 400 configurations, is also reported. Clustering of substitutions (left side) results in a decrease in the ionic density near the surface due to ion-ion repulsion.

A difference between $\mathrm{NaCl}$ and $\mathrm{CsCl}$ salts is also observed in the case of the uncharged pyrophyllite. Specifically, the strongly hydrated $\mathrm{Na}^{+}$cations are somewhat depleted from the first adsorbed water layers. In contrast, an excess of $\mathrm{Cs}^{+}$is seen near the surface. Even though $\mathrm{Cs}^{+}$remains solvated, this is reminiscent of the behaviour observed at the liquidvapour interface of water. ${ }^{54}$ This further supports the hydrophobic behaviour of the neutral pyrophyllite surface, which was already confirmed in molecular simulation in particular by 
the computation of contact angles. ${ }^{55}$

\subsubsection{Water diffusion}

The nature of the ion and of the clay mineral also has an influence on the dynamics of the adsorbed fluid. Specifically, we discuss here the component $D_{\|}$of the diffusion tensor of water along the clay surfaces. Since it depends on the distance of the water molecules from the surface, we first define relevant regions in which $D_{\|}$is computed, based on the density profiles. ${ }^{31}$ These regions, illustrated by vertical lines in Figure 4, are defined according to the position $z$ of the water oxygen atoms: within $4 \AA$ of the plane containing the surface oxygen atoms, between 4 and $7 \AA$, between 7 and $10 \AA$; the central region has a width of $30.8 \AA . \mathrm{D}_{\|}$is then calculated in each layer as explained in Ref. 31 .

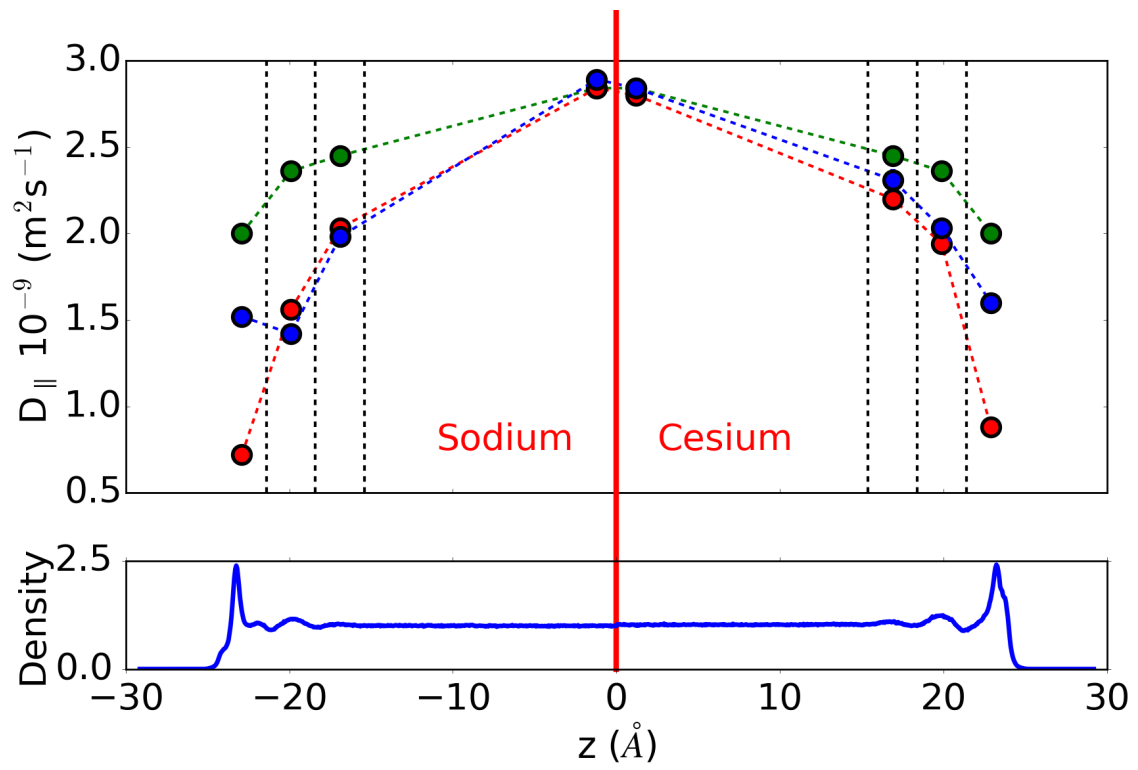

Figure 4: Water diffusion coefficient along the surface, $D_{\|}$, in the regions defined by the dashed vertical lines, for the three different clays (green for pyrophyllite, blue for montmorillonite and red for beidellite) and the two cations $\left(\mathrm{Na}^{+}\right.$left, $\mathrm{Cs}^{+}$right), in the absence of added salt. The corresponding total density profiles are also shown in the bottom panel to clarify the definition of the various regions, in the case of montmorillonite. Error bars are smaller than the symbols.

The results are presented in Figure 4 for both cations in the absence of added salt (note that the system is the same for pyrophyllite). In the bulk region, we obtain the same 
diffusion coefficient for all surfaces, as expected. The common value of $2.910^{-9} \mathrm{~m}^{2} \mathrm{~s}^{-1}$ is larger than the experimental value for bulk water $\left(2.310^{-9} \mathrm{~m}^{2} \mathrm{~s}^{-1}\right)$. Two factors may explain such a discrepancy. On the one hand, the SPC/E water model is known to overestimate the bulk diffusion coefficient (the extrapolated value for an infinite cubic box size is close to $3.010^{-9} \mathrm{~m}^{2} \mathrm{~s}^{-1}$, see Ref. 56). On the other hand, the diffusion coefficient computed by molecular dynamics depends on the size and the shape of the simulation box, due to hydrodynamic finite-size effects. We derived in Ref. 57 an analytical expression for the correction $\Delta D_{\|}$to the average diffusion coefficient along planar walls separated by a distance $H$, with stick boundary conditions, due to the finite length $L$ of the simulation box in the directions along the walls. Even though in the present case the box lengths in the $x$ and $y$ directions are not equal, this is approximately the case. When $H>L$ (elongated systems), the correction reads:

$$
\Delta D_{\|}(H>L)=\frac{k_{B} T}{\eta}\left[\frac{3}{40} \frac{H}{L^{2}}-\frac{3 \ln (1+\sqrt{2})}{4 \pi L}\right]
$$

which was shown to be in very good agreement with molecular dynamics simulation results for a Lennard-Jones fluid between walls of Lennard-Jones particles. In the present case, this expression leads to corrections ranging from $-1.1 \times 10^{-10}$ to $-1.7 \times 10^{-10} \mathrm{~m}^{2} \mathrm{~s}^{-1}$ for all systems. These corrections are smaller than most variations of the diffusion coefficients presented below to discuss the effects of the nature of the surface and counterions (see the results of Figure 4).

In all cases, $D_{\|}$decreases as water approaches the surface (the only exception, discussed below, is the first water layer on Na-montmorillonite, which diffuses slightly faster than the second layer). For both counterions, the presence of substitutions in the mineral results in smaller water diffusion coefficients near the surface, possibly due to the change in orientation of molecules due to the surface electric field, ${ }^{39}$ but also to the associated presence of counterions in the adsorbed water layers. Indeed, the residence of water in the solvation shell 
of cations tends to slow down their overall diffusion. Within the first layer, $D_{\|}$is reduced more dramatically for both counterions on the surface of beidellite than that of montmorillonite. This can be explained by the localization of the substitutions closer to the fluid in the former case, resulting in a stronger interaction of the cations with the surface. Overall, water diffuses faster with $\mathrm{Cs}^{+}$as a counterion on both beidellite and montmorillonite. This subtle combination of surface- and ion-specific effects can be explained by the larger density of $\mathrm{Na}^{+}$in that layer compared to $\mathrm{Cs}^{+}$as discussed in the previous section (the density profiles of Figure 2 correspond to the case with added salt, but the results are similar without salt). The virtual absence of inner-sphere complexes on Na-montmorillonite then explains the non-monotonic behaviour of $D_{\|}$in the vicinity of that surface. Similar observations can be made in the case of added salt (results not shown). One observes however a global decrease of $D_{\|}$in that case. In the central regions, the computed diffusion coefficients are 2.5 and $2.710^{-9} \mathrm{~m}^{2} \mathrm{~s}^{-1}$ for $\mathrm{Na}^{+}$and $\mathrm{Cs}^{+}$, respectively. A larger decrease in the former case is consistent with previous simulations ${ }^{58}$ and with experiments ${ }^{59}$ for bulk electrolyte solutions.

The reported results for beidellite are averages over both surfaces. As explained above, the ion density profiles are in fact asymmetric due to the different distribution of the substitutions within the tetrahedral layers (see Figure 3). The difference in cation distributions also results in an asymmetry in the diffusion coefficients in the first (and to a lesser extent second) adsorbed layer. For example, in the $\mathrm{Na}^{+}$case without added salt we obtain $(0.76 \pm 0.02)$ and $(0.70 \pm 0.01) 10^{-9} \mathrm{~m}^{2} \mathrm{~s}^{-1}$ in the first adsorbed layer on the left and right surfaces, respectively. The asymmetry is less pronounced with added salt, with $(0.72 \pm 0.01)$ vs $(0.68 \pm 0.02) 10^{-9} \mathrm{~m}^{2} \mathrm{~s}^{-1}$.

To conclude this first part on the equilibrium properties, the present results indicate that the charge of the clay surface and its localization within the mineral layer has no effect on water diffusion away from the first few molecular layers, but it does have a significant impact within the latter. In addition, the nature of the cation has an influence on the diffusion of water within the first adsorbed layer. These features can be explained by the mineral- and 
ion-specific structure of the interface.

\subsection{Hydrodynamics: slip, stick and stagnation}

\subsubsection{Continuous hydrodynamics}

We now turn to the hydrodynamic properties of these interfaces. Following previous studies, we apply a body force on water molecules and ions and measure the steady-state velocity profile. At the continuum level, the balance between viscous and other forces is then described by Stokes equation $\eta \Delta \mathbf{v}+\mathbf{f}_{V}=\mathbf{0}$, where $\eta$ is the fluid viscosity, $\mathbf{v}$ is the velocity and $\mathbf{f}_{V}$ the volumic force acting on the fluid. In the case of the Poiseuille flow (uniform $\mathbf{f}_{V}=\mathrm{f}_{V} \mathbf{e}_{\mathbf{x}}$ in a slit pore), the profile $\mathbf{v}=v(z) \mathbf{e}_{\mathbf{x}}$ is parabolic. Defining $z=0$ as the mid-plane of the pore and $\pm \frac{L_{h y d}}{2}$ the position of the hydrodynamic interfaces, $v(z)$ reads:

$$
v(z)=v\left(\frac{L_{h y d}}{2}\right)-\frac{f_{V}}{2 \eta}\left[z^{2}-\left(\frac{L_{h y d}}{2}\right)^{2}\right]
$$

Therefore the viscosity $\eta$ can be computed from the curvature of the profile and the velocity at the interface $v\left(L_{h y d} / 2\right)$ can be used to determine appropriate boundary conditions. When the velocity of the fluid does not vanish at the interface (slip boundary condition), a slip length $b$ can be defined as: ${ }^{5}$

$$
\frac{1}{b}=-\frac{1}{v\left(L_{h y d} / 2\right)}\left(\frac{\mathrm{d} v}{\mathrm{~d} z}\right)_{z=L_{h y d} / 2} .
$$

It can therfore be determined from the molecular simulation velocity profile when $L_{h y d}$ is known. Several choices are possible. In particular, Bocquet and Barrat provided a GreenKubo expression for the location of the "hydrodynamic position". ${ }^{11}$ Following previous work, ${ }^{37,39}$ we consider here a more phenomenolgical approach, based on the partitioning of the pore space between a region of homogeneous fluid and a region without fluid. Therefore we identify in this slip case the position of the hydrodynamic interfaces $\pm L_{h y d} / 2$ with 
the positions $\pm z_{G D S}$ of the Gibbs Dividing Surfaces (GDS):

$$
\int_{0}^{ \pm z_{G D S}}\left[\rho_{\text {bulk }}-\rho(z)\right] \mathrm{d} z=\int_{ \pm z_{G D S}}^{+\infty} \rho(z) \mathrm{d} z
$$

where $\rho_{\text {bulk }}$ and $\rho(z)$ are respectively the fluid density in the bulk, i.e. in the central region of the pore, and that at $z$. Thus $z_{G D S}$ can be calculated from the density profile of the fluid $\rho(z)$ obtained by simulation (see Figure 5). In contrast, when the fluid sticks to the surfaces, a layer of thickness $d$ is immobile. The positions of the shear planes can be determined from the MD velocity profiles as $v\left(L_{h y d} / 2\right)=0$. In this stick case they are located further from the surface than the GDS. The distance between the shear plane and the GDS then defines a stagnation length $d$ as:

$$
d=z_{G D S}-\frac{L_{h y d}}{2}
$$

The location of the GDS and the hydrodynamic width for all systems will be reported below, together with the corresponding slip or stagnation lengths.

As can be seen on Figure 5, the parabolic fit in the central region is a very good approximation, even if a common value of the viscosity is used for similar systems (see next section). It should be noted, however, that deviations from the parabolic profile may be observed near the wall where the fluid is layered. The resulting estimate of the flux near the wall by the parabolic profile is therefore inaccurate, but this contribution to the overall flux is small - even more so that the pore is larger. ${ }^{37}$ It is more important to accurately describe the profile further from the surface by introducing an appropriate boundary condition.

\subsubsection{Viscosity of the confined fluid}

A force of 0.75 cal.mol $^{-1} \AA^{-1}$ per atom is applied in the $x$ direction. This value was chosen in the range where the response of the system is linear. The steady-state velocity profiles for the three clays and both cations, are reported in Figure 6 without and with added salt in panels $6 \mathrm{a}$ and $6 \mathrm{~b}$, respectively. The nature of the clay, the nature of the counterion and the 


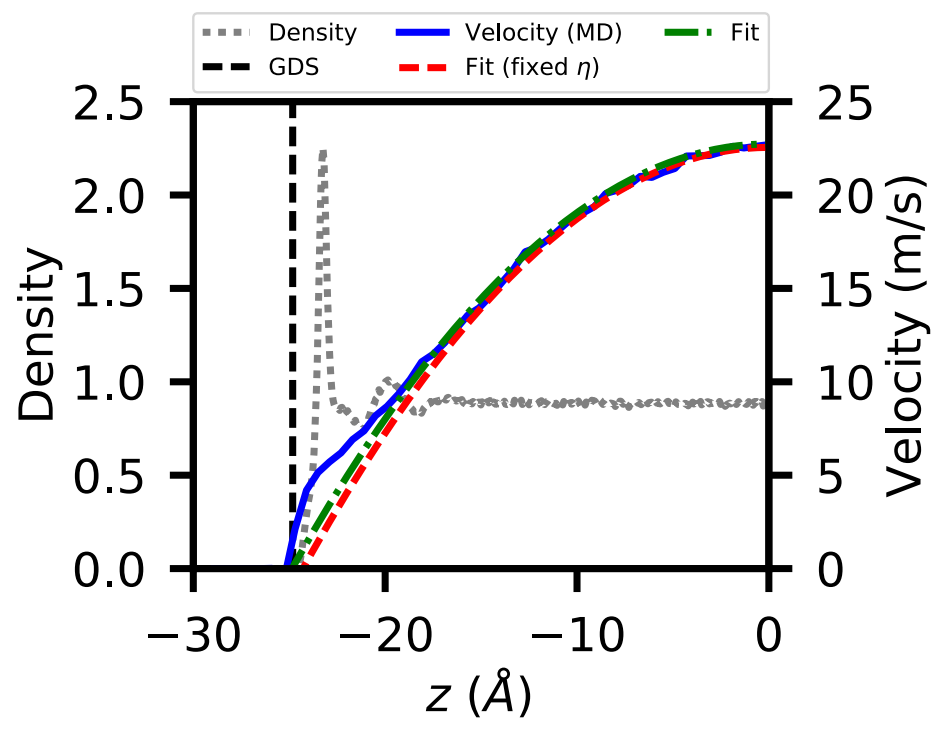

Figure 5: The velocity profile obtained by non-equilibrium molecular dynamics (solid blue line) here for montmorillonite with $\mathrm{Na}^{+}$as counterion without added salt, is fitted to a parabola in the central region (green dashed-dotted line) and the viscosity is determined from the curvature. The figure also shows the fit to a parabola enforcing a value of the viscosity common for similar systems (see text, red dashed line), which is then used to determine the boundary conditions. The Gibbs Dividing Surfaces (GDS, vertical black dashed line, defined by Eq. 4), determined from the density profile (grey dotted line) is used to localize the solid/fluid interface.

salt concentration all contribute to the hydrodynamic response. Nevertheless, in all cases the velocity profile is parabolic in the central region, so that the viscosity can be computed from the curvature (in practice, the fit is done in a central slab of width $3 \mathrm{~nm}$ ). The corresponding values are summarized in Table 1. The reported error bars on the viscosity were obtained by comparing the estimates for the first and second half of the production runs.

Despite some variations, the results for a given family of systems are similar. In the saltfree case, regardless of the nature of counterion, the values are consistent with our previous work on pyrophyllite and Na-montmorillonite. ${ }^{37,39}$ In the following analysis of the velocity profile, we therefore use the average value in all salt-free systems which is in perfect agreement with the bulk value $(\eta=0.68 \mathrm{cP})$ for the $\mathrm{SPC} / \mathrm{E}$ water model at room temperature. ${ }^{56}$ Similarly, the viscosities for a given salt are consistent for all clays and in the following we use a common value of $0.89 \mathrm{cP}$ (resp. $0.78 \mathrm{cP}$ ) for all systems with $1 \mathrm{M}$ of $\mathrm{NaCl}$ (resp 

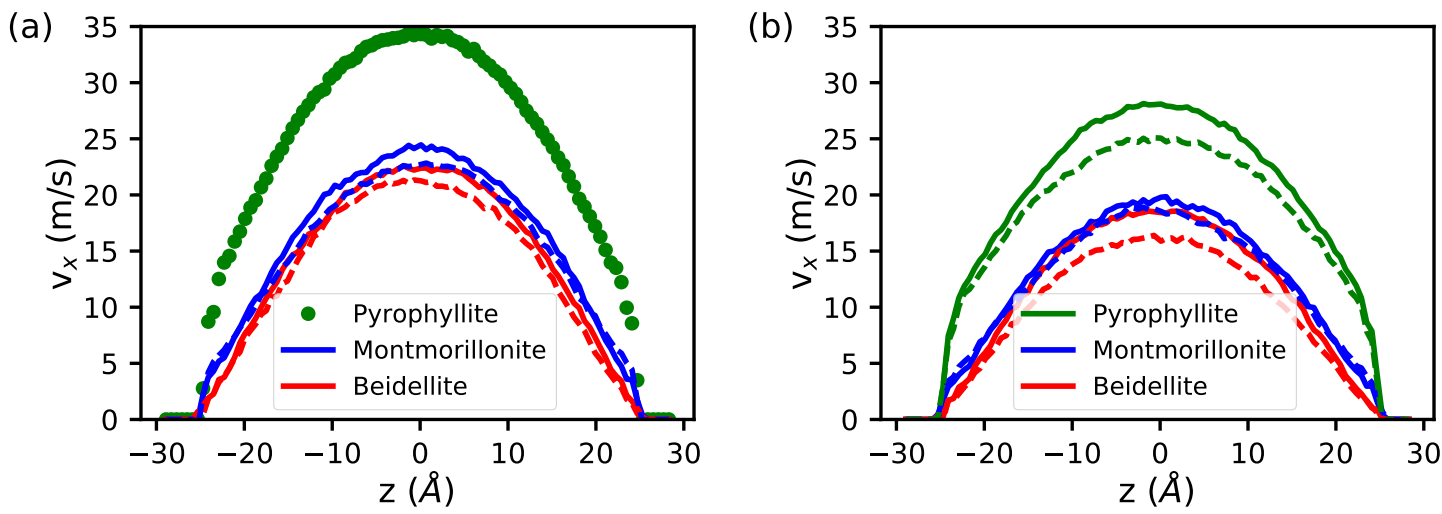

Figure 6: Steady-state velocity profiles under an applied force parallel to the surface applied to each fluid atom (the same force is applied in all cases), in the absence of added salt (a) and with $1 \mathrm{M}$ of salt (b). Dashed and solid lines correspond to $\mathrm{Na}^{+}$and $\mathrm{Cs}^{+}$counterions, respectively, while colors refer to the clay type: green for pyrophyllite, blue for montmorillonite and red for beidellite. Note that in (a) no cations are present for pyrophyllite, so that symbols are used instead of lines.

$\mathrm{CsCl}$ ). An increase in the viscosity with $\mathrm{NaCl}$ concentration is consistent with experimental results. ${ }^{60}$ In contrast, the less pronounced increase in the $\mathrm{CsCl}$ case points to a limitation of the force field, since the experimental viscosity decreases in the presence of $\mathrm{CsCl}$ salt. ${ }^{61}$ Nevertheless, the smaller viscosity with $\mathrm{Cs}^{+}$compared to $\mathrm{Na}^{+}$is in the same order as in the experiments. We finally note that the choice of common values to model all velocity profiles of systems without salt, with $1 \mathrm{M}$ of $\mathrm{NaCl}$ or $\mathrm{CsCl}$, even though it does not change the conclusions of the discussion below, may have a quantitative influence on the resulting slip and stagnation length and therefore contributes to the reported uncertainties.

\subsubsection{Hydrodynamic boundary conditions}

From the velocity profiles, and under the above assumptions on where to apply the boundary condition and on the value of the viscosity, we determine in each case the slip length $b$ or the stagnation length $d$. The results are summarized in Tables 2 and 3 for $\mathrm{Na}^{+}$and $\mathrm{Cs}^{+}$, respectively, together with the corresponding location of the GDS and hydrodynamic width. Reported uncertainties are based on comparing estimates for two half trajectories as well as 
Table 1: Fluid viscosities for the three clays with $\mathrm{Na}^{+}$or $\mathrm{Cs}^{+}$as counterion and two salt concentrations.

\begin{tabular}{|c|c|c|}
\hline Clay & Salt & Viscosity (cP) \\
\hline Pyro & - & $0.62 \pm 0.03$ \\
Pyro & $\mathrm{NaCl} 1 \mathrm{M}$ & $0.95 \pm 0.02$ \\
Pyro & $\mathrm{CsCl} 1 \mathrm{M}$ & $0.74 \pm 0.02$ \\
\hline Mont-Na & - & $0.70 \pm 0.02$ \\
Mont-Cs & - & $0.71 \pm 0.02$ \\
Mont-Na & $\mathrm{NaCl} 1 \mathrm{M}$ & $0.84 \pm 0.02$ \\
Mont-Cs & $\mathrm{CsCl} 1 \mathrm{M}$ & $0.79 \pm 0.06$ \\
\hline Beid-Na & - & $0.74 \pm 0.02$ \\
Beid-Cs & - & $0.68 \pm 0.03$ \\
Beid-Na & $\mathrm{NaCl} 1 \mathrm{M}$ & $0.88 \pm 0.02$ \\
Beid-Cs & $\mathrm{CsCl} 1 \mathrm{M}$ & $0.80 \pm 0.04$ \\
\hline
\end{tabular}

the choice of viscosity (see previous section).

Table 2: Position of the Gibbs dividing surface $z_{G D S}$, Slip length $b$ or stagnation length $d$ of the fluid in $\AA$, for the three $\mathrm{Na}$-clays and two $\mathrm{NaCl}$ concentrations. The boundary condition is determined from the velocity profile using a viscosity of $0.68 \mathrm{cP}$ for without added salt and $0.89 \mathrm{cP}$ with $1 \mathrm{M} \mathrm{NaCl}$.

\begin{tabular}{|c|c|c|c|c|c|}
\hline Clay & NaCl salt & $z_{G D S}(\AA)$ & $L_{h y d} / 2(\AA)$ & $b(\AA)$ & $d(\AA)$ \\
\hline Pyro & $0 \mathrm{M}$ & $24.2 \pm 0.2$ & $24.2 \pm 0.2$ & $5.9 \pm 0.4$ & - \\
Pyro & $1 \mathrm{M}$ & $24.1 \pm 0.2$ & $24.1 \pm 0.2$ & $5.6 \pm 0.3$ & - \\
\hline Mont-Na & $0 \mathrm{M}$ & $24.8 \pm 0.2$ & $24.3 \pm 0.5$ & - & $0.5 \pm 0.3$ \\
Mont-Na & $1 \mathrm{M}$ & $24.9 \pm 0.2$ & $24.9 \pm 0.2$ & $0.2 \pm 0.5$ & - \\
\hline Beid-Na & $0 \mathrm{M}$ & $24.8 \pm 0.2$ & $23.6 \pm 0.5$ & - & $1.2 \pm 0.3$ \\
Beid-Na & $1 \mathrm{M}$ & $24.6 \pm 0.2$ & $23.7 \pm 0.6$ & - & $0.9 \pm 0.5$ \\
\hline
\end{tabular}

Effect of surface charge and its localization With both cations, with and without added salt, we observe a clear pattern of decreasing slip from the neutral pyrophyllite to the charged montmorillonite (with octahedral substitutions) and beidellite (with tetrahedral substitutions). More precisely, a slip boundary condition with a slip length of approximately two water layers applies to pyrophyllite; a stick boundary condition applies to montmorillonite with $b \approx 0$ or $d \approx 0$ (except with $1 \mathrm{M} \mathrm{CsCl}$ ); a small stagnation layer of approximately one half of water diameter should be introduced for beidellite. In a previous study of Na- 
Table 3: Slip length $b$ or stagnation length $d$ of the fluid in $\AA$, for the three Cs-clays and two $\mathrm{CsCl}$ concentrations. The boundary condition is determined from the velocity profile using a viscosity of $0.68 \mathrm{cP}$ for without added salt and $0.78 \mathrm{cP}$ with $1 \mathrm{M} \mathrm{CsCl}$.

\begin{tabular}{|c|c|c|c|c|c|}
\hline Clay & CsCl salt & $z_{G D S}(\AA)$ & $L_{h y d} / 2(\AA)$ & $b(\AA)$ & $d(\AA)$ \\
\hline Pyro & $0 \mathrm{M}$ & $24.3 \pm 0.2$ & $24.3 \pm 0.2$ & $5.9 \pm 0.4$ & - \\
Pyro & $1 \mathrm{M}$ & $24.1 \pm 0.2$ & $24.1 \pm 0.2$ & $5.2 \pm 0.4$ & - \\
\hline Mont-Cs & $0 \mathrm{M}$ & $25.3 \pm 0.2$ & $25.0 \pm 0.5$ & - & $0.3 \pm 0.3$ \\
Mont-Cs & $1 \mathrm{M}$ & $25.3 \pm 0.2$ & $24.2 \pm 0.6$ & - & $1.1 \pm 0.4$ \\
\hline Beid-Cs & $0 \mathrm{M}$ & $25.5 \pm 0.2$ & $24.2 \pm 0.6$ & - & $1.3 \pm 0.4$ \\
Beid-Cs & $1 \mathrm{M}$ & $25.5 \pm 0.2$ & $23.8 \pm 0.6$ & - & $1.7 \pm 0.4$ \\
\hline
\end{tabular}

Montmorillonite with the CLAYFF force field, we had determined a slip boundary condition with a slip length of $b=1.4 \pm 0.3 \AA$. Here we find instead a small stagnation length $d=0.5 \pm 0.3 \AA$. While such a difference is not huge, it is statistically significant and may be due to the fact that in the former study the clay layers were treated as rigid, while in the present work we account for their flexibility and their finite temperature. It is therefore not surprising that the resulting energy dissipation at the wall results in less slippage at the interface. However the effect of wall flexibility on the flow is not straightforward, as recent work on carbon nanotubes suggests e.g. that it could reduce the friction at the interface, ${ }^{62}$ and coupling of the fluid dynamics with phonons in such tubes may significantly enhance the diffusion of water. ${ }^{63,64}$ We also note that the difference between neutral and charged surfaces is much larger than between the two charged clays. Nevertheless, the small stagnation length in the case of beidellite (compared to $b \approx 0$ or $d \approx 0$ for montmorillonite) reflects the stronger interaction of cations with the substitutions which are closer to the fluid in that case, as explained above.

The effect of surface charge on hydrodynamic slippage was analyzed quantitatively by Joly et al. ${ }^{65}$ More precisely, they predicted and confirmed by MD simulations for a LennardJones (LJ) fluid with LJ walls that the slip length varies $b=b_{L J} /\left[1+(1 / \alpha)\left(\Sigma \sigma^{2} / e\right)^{2}\left(l_{B} / \sigma\right) b_{L J}\right]$ with $b_{L J}$ the slip length for a neutral surface, $\alpha \approx 1$ a constant, $\Sigma$ the surface charge density, $\sigma$ the LJ diameter of the wall atoms and $l_{B}$ the Bjerrum length. This simple expression 
correctly predicts the decrease in $b$ from neutral pyrophyllite to charged montmorillonite and beidellite, even though it is not sufficient to quantatively describe the subtle difference between the latter two.

Effect of salt concentration and ion specificity In the absence of added salt, the results are very similar for both counterions. There is little effect of adding $\mathrm{NaCl}$ salt, except for a slight decrease in the slip length for pyrophyllite. Adding $\mathrm{CsCl}$ slightly increases the stagnation length with montmorillonite and beidellite and slightly decreases the slip length with pyrophyllite. Overall, the effects of added salt and of the nature of the counterions are less pronounced than that of the surface charge and its localization.

As a final remark, we note that the above discussion of hydrodynamic boundary conditions is based on a parabolic fit of the flow profile in the central region of the pore. While this is the most relevant from a practical point of view in order to introduce mineral- and ionspecificity into hydrodynamic descriptions for larger scales, it should be reminded that the molecular velocity profile deviates from this parabolic fit in the close vicinity of the surface. This is obvious in Figure 5, where the velocity in the first adsorbed layer is larger than the profile extrapolated from the bulk. In this region, the shape of the molecular velocity profile may also be different from what would be inferred from the bulk viscosity. While introducing a local viscosity to account for such variations is not sufficient to obtain a quantitative description, ${ }^{37}$ an illustration of such an effect can be found in Figure 6a with the case of montmorillonite. In this salt-free case, the velocity increases more slowly close to the surface with $\mathrm{Na}^{+}$as a counterion than with $\mathrm{Cs}^{+}$, even though the fluid far from the surface is the same in both cases (hence identical viscosities). Such a difference probably arises because fully hydrated $\mathrm{Na}^{+}$near the surface (outer-sphere complexes) have a stronger effect on the interfacial fluid dynamics than $\mathrm{Cs}^{+}$ions which form inner-sphere complexes. 


\section{Conclusion}

The structure, diffusion and hydrodynamic properties of clay interfaces with aqueous solution depends on the nature of the clay, the nature of the counterions and the salt concentration in the solution. We used molecular dynamics to investigate water-filled nanopores between uncharged (pyrophyllite) and charged (montmorillonite and beidellite, with susbtitutions located in the octahedral and tetrahedral layers, respectively) clays, with sodium or cesium as counterions, in the absence and in the presence of added salt. We discussed how the balance between solvation and attraction of the cations to the surface results in various distributions between inner- and outer-sphere complexes and how this influences the dynamics of water near the surface, as well as the hydrodynamic flow in the presence of an external force. In the latter case, the discussion based on a mapping of the molecular velocity profiles to a continuous description (parabolic Poiseuille flow) shows that the larger effects come from the presence/absence of charge in the mineral, as well as the localization of substitutions within the clay layer. The salt concentration and the nature of the counterions have a comparatively less important impact far from the surface - even though some differences are observed in its close vicinity, which are not properly captured by the continuous description.

In the future, this study could benefit from recent developments of improved force fields which allow to better capture ion- and mineral-specificity in clays and related aluminosilicate minerals, ${ }^{66-68}$ starting with the structure and diffusion under equlibrium conditions. Such models may also improve the description of the structural relaxation of the mineral itself, induced by the truncation of the mineral periodicity at the interface with the liquid, demonstrated e.g. in X-ray reflectivity experiments on mica. ${ }^{69}$ This work could also be extended using force fields which better describe concentrated electrolyte solutions, e.g. via rescaled charges ${ }^{70}$ or introducing polarization effects, ${ }^{71}$ as well as by considering other ions, includ-

ing divalent ones, and of course other simple or more complex including binary fluids. ${ }^{72-75}$ As a first step, one could for example examine mixtures of sodium and cesium counterions. Finally, in the broader perspective of transport on the macroscopic scale, the results of the 
present work could be introduced in a bottom-up approach combining molecular simulation results with transport models on larger scales. ${ }^{76-78}$

\section{Acknowledgement}

The authors acknowledge financial support from IFPEN and access to computing resources on Curie (TGCC, French National HPC) via the GENCI project x2016087684 and on MeSU (UPMC).

\section{References}

(1) Siria, A.; Bocquet, M.-L.; Bocquet, L. Nature Reviews Chemistry 2017, 1, 91.

(2) Aguilera, R. SPE Reservoir Evaluation E Engineering 2014, 17, 190-208.

(3) Gaus, I. International Journal of Greenhouse Gas Control 2010, 4, 73-89.

(4) Altmann, S.; Tournassat, C.; Goutelard, F.; Parneix, J.-C.; Gimmi, T.; Maes, N. Applied Geochemistry 2012, 27, 463-478.

(5) Bocquet, L.; Charlaix, E. Chemical Society Reviews 2010, 39, 1073-1095.

(6) Falk, K.; Coasne, B.; Pellenq, R.; Ulm, F.-J.; Bocquet, L. Nature Communications 2015, 6, 6949.

(7) Hoang, H.; Galliero, G. Physical Review E 2012, 86, 021202.

(8) Hoang, H.; Galliero, G. The Journal of Chemical Physics 2012, 136, 124902-124902-8.

(9) Hoang, H.; Galliero, G. The Journal of Chemical Physics 2012, 136, 184702-18470211.

(10) Hoang, H.; Galliero, G. The Journal of Chemical Physics 2013, 138, 054707-05470711. 
(11) Bocquet, L.; Barrat, J.-L. Physical Review E 1994, 49, 3079-3092.

(12) Bocquet, L.; Barrat, J. L. Journal of Physics: Condensed Matter 1996, 8, 9297.

(13) Chen, J.; Yu, H.; Fan, J.; Wang, F.; Lu, D.; Liu, H.; Wu, H. AIP Advances 2017, 7, 045217.

(14) Huang, D. M.; Sendner, C.; Horinek, D.; Netz, R. R.; Bocquet, L. Physical Review Letters 2008, 101, 226101.

(15) Cygan, R. T.; Liang, J.-J.; Kalinichev, A. G. The Journal of Physical Chemistry B 2004, 108, 1255-1266.

(16) Wang, J.; Kalinichev, A. G.; Kirkpatrick, R. J. Geochimica et Cosmochimica Acta 2006, 70, 562-582.

(17) Kalinichev, A. G.; Wang, J.; Kirkpatrick, R. J. Cement and Concrete Research 2007, 37, 337-347.

(18) Argyris, D.; Cole, D. R.; Striolo, A. ACS Nano 2010, 4, 2035-2042.

(19) Dongshuai, H.; Zeyu, L.; Peng, Z.; Qingjun, D. Physical Chemistry Chemical Physics 2016, 18, 2059-2069.

(20) Loganathan, N.; Yazaydin, A. O.; Bowers, G. M.; Kalinichev, A. G.; Kirkpatrick, R. J. The Journal of Physical Chemistry C 2016, 120, 10298-10310.

(21) Boţan, A.; Rotenberg, B.; Marry, V.; Turq, P.; Noetinger, B. The Journal of Physical Chemistry C 2010, 114, 14962-14969.

(22) Morrow, C. P.; Yazaydin, A. O.; Krishnan, M.; Bowers, G. M.; Kalinichev, A. G.; Kirkpatrick, R. J. The Journal of Physical Chemistry C 2013, 117, 5172-5187.

(23) Ngouana W., B. F.; Kalinichev, A. G. The Journal of Physical Chemistry C 2014, 118, 12758-12773. 
(24) Greathouse, J. A.; Cygan, R. T.; Fredrich, J. T.; Jerauld, G. R. The Journal of Physical Chemistry C 2016, 120, 1640-1649.

(25) Loganathan, N.; Yazaydin, A. O.; Bowers, G. M.; Kalinichev, A. G.; Kirkpatrick, R. J. The Journal of Physical Chemistry C 2017, 121, 24527-24540.

(26) Loganathan, N.; Bowers, G. M.; Yazaydin, A. O.; Schaef, H. T.; Loring, J. S.; Kalinichev, A. G.; Kirkpatrick, R. J. The Journal of Physical Chemistry C 2018, 122, 4391-4402.

(27) Greathouse, J. A.; Cygan, R. T. Physical Chemistry Chemical Physics 2005, 7, 3580.

(28) Greathouse, J. A.; Cygan, R. T. Environmental Science $\&$ Technology 2006, 40, 38653871.

(29) Tournassat, C.; Chapron, Y.; Leroy, P.; Bizi, M.; Boulahya, F. Journal of Colloid and Interface Science 2009, 339, 533-541.

(30) Bourg, I. C.; Lee, S. S.; Fenter, P.; Tournassat, C. The Journal of Physical Chemistry C 2017, 121, 9402-9412.

(31) Marry, V.; Rotenberg, B.; Turq, P. Phys. Chem. Chem. Phys. 2008, 10, 4802-4813.

(32) Rotenberg, B.; Marry, V.; Malikova, N.; Turq, P. Journal of Physics: Condensed Matter 2010, 22, 284114.

(33) Bourg, I. C.; Sposito, G. Journal of Colloid and Interface Science 2011, 360, 701-715.

(34) Rotenberg, B. MRS Bulletin 2014, 39, 1074-1081.

(35) Greathouse, J. A.; Hart, D. B.; Bowers, G. M.; Kirkpatrick, R. J.; Cygan, R. T. The Journal of Physical Chemistry C 2015, 119, 17126-17136.

(36) Bacle, P.; Dufrêche, J.-F.; Rotenberg, B.; Bourg, I. C.; Marry, V. Applied Clay Science 2016, 123, 18-28. 
(37) Boţan, A.; Rotenberg, B.; Marry, V.; Turq, P.; Noetinger, B. The Journal of Physical Chemistry C 2011, 115, 16109-16115.

(38) Dufrêche, J. F.; Marry, V.; Malikova, N.; Turq, P. Journal of Molecular Liquids 2005, $118,145-153$.

(39) Boţan, A.; Marry, V.; Rotenberg, B.; Turq, P.; Noetinger, B. The Journal of Physical Chemistry C 2013, 117, 978-985.

(40) Boţan, A.; Marry, V.; Rotenberg, B.; Turq, P.; Noetinger, B. The Journal of Physical Chemistry C 2013, 117, 20376-20376.

(41) Rotenberg, B.; Pagonabarraga, I. Molecular Physics 2013, 111, 827-842.

(42) Bhadauria, R.; Aluru, N. R. The Journal of Chemical Physics 2017, 146, 184106.

(43) Teich-McGoldrick, S. L.; Greathouse, J. A.; Jové-Colón, C. F.; Cygan, R. T. The Journal of Physical Chemistry C 2015, 119, 20880-20891.

(44) Chávez-Páez, M.; Workum, K. V.; Pablo, L. d.; Pablo, J. J. d. The Journal of Chemical Physics 2001, 114, 1405.

(45) Berendsen, H. J. C.; Grigera, J. R.; Straatsma, T. P. Journal of Physical Chemistry 1987, 91, 6269-6271.

(46) Dang, L. X. The Journal of Physical Chemistry B 2002, 106, 10388-10394.

(47) Frenkel, D.; Smit, B. Understanding molecular simulation: from algorithms to applications, 2d edition; Academic Press: London, 2002.

(48) Ryckaert, J.-P.; Ciccotti, G.; Berendsen, H. J. C. Journal of Computational Physics 1977, 23, 327-341.

(49) Hockney, R. W.; Eastwood, J. W. Computer simulation using particles; Hilger: Bristol, 1988. 
(50) Martyna, G. J.; Klein, M. L.; Tuckerman, M. The Journal of Chemical Physics 1992, $97,2635-2643$.

(51) Wang, J.; Kalinichev, A. G.; Kirkpatrick, R. J. The Journal of Physical Chemistry C 2009, 113, 11077-11085.

(52) Sposito, G.; Skipper, N. T.; Sutton, R.; Park, S.-h.; Soper, A. K.; Greathouse, J. A. Proceedings of the National Academy of Sciences 1999, 96, 3358-3364.

(53) Rotenberg, B.; Morel, J. P.; Marry, V.; Turq, P.; Morel-Desrosiers, N. Geochimica et Cosmochimica Acta 2009, 73, 4034-4044.

(54) Jungwirth, P.; Tobias, D. J. Chemical Reviews-Columbus 2006, 106, 1259-1281.

(55) Rotenberg, B.; Patel, A. J.; Chandler, D. Journal of the American Chemical Society 2011, 133, 20521-20527.

(56) Tazi, S.; Boțan, A.; Salanne, M.; Marry, V.; Turq, P.; Rotenberg, B. Journal of Physics: Condensed Matter 2012, 24, 284117.

(57) Simonnin, P.; Noetinger, B.; Nieto-Draghi, C.; Marry, V.; Rotenberg, B. Journal of Chemical Theory and Computation 2017, 13, 2881-2889.

(58) Hartkamp, R.; Coasne, B. The Journal of Chemical Physics 2014, 141, 124508.

(59) Mills, R.; Lobo, V. Self-diffusion in Electrolyte Solutions; Physical Sciences Data 36; Elsevier, 1989.

(60) Banipal, T. S.; Singh, H.; Banipal, P. K. Thermochimica Acta 2013, 572, 6-22.

(61) Toshiaki, I. Reports of the Institute of Physical and Chemical Research 1985, 61, 53-79.

(62) Sam, A.; Kannam, S. K.; Hartkamp, R.; Sathian, S. P. The Journal of Chemical Physics 2017, 146, 234701. 
(63) Ma, M.; Grey, F.; Shen, L.; Urbakh, M.; Wu, S.; Liu, J. Z.; Liu, Y.; Zheng, Q. Nature Nanotechnology 2015, 10, 692-695.

(64) Bocquet, L.; Netz, R. R. Nature Nanotechnology 2015, 10, 657-658.

(65) Joly, L.; Ybert, C.; Trizac, E.; Bocquet, L. The Journal of Chemical Physics 2006, 125, 204716-204716-14.

(66) Tesson, S.; Salanne, M.; Rotenberg, B.; Tazi, S.; Marry, V. The Journal of Physical Chemistry C 2016, 120, 3749-3758.

(67) Tesson, S.; Louisfrema, W.; Salanne, M.; Boutin, A.; Rotenberg, B.; Marry, V. The Journal of Physical Chemistry C 2017, 121, 9833-9846.

(68) Hånde, R.; Ramothe, V.; Tesson, S.; Dazas, B.; Ferrage, E.; Lanson, B.; Salanne, M.; Rotenberg, B.; Marry, V. Minerals 2018, 8, 205.

(69) Lee, S. S.; Fenter, P.; Nagy, K. L.; Sturchio, N. C. Langmuir 2012, 28, 8637-8650.

(70) Kann, Z. R.; Skinner, J. L. The Journal of Chemical Physics 2014, 141, 104507.

(71) Tazi, S.; Molina, J. J.; Rotenberg, B.; Turq, P.; Vuilleumier, R.; Salanne, M. The Journal of Chemical Physics 2012, 136, 114507.

(72) Ameur, D.; Galliero, G. Microfluidics and Nanofluidics 2013, 15, 183-189.

(73) Phan, A.; Cole, D. R.; Wei, R. G.; Dzubiella, J.; Striolo, A. ACS Nano 2016, 10, $7646-7656$.

(74) Zhang, L.; Lu, X.; Liu, X.; Yang, K.; Zhou, H. Energy 83 Fuels 2016, 30, 149-160.

(75) He, S.; Palmer, J. C.; Qin, G. Microporous and Mesoporous Materials 2017, 249, 88-96.

(76) Obliger, A.; Duvail, M.; Jardat, M.; Coelho, D.; Békri, S.; Rotenberg, B. Physical Review E 2013, 88, 013019. 
(77) Obliger, A.; Jardat, M.; Coelho, D.; Békri, S.; Rotenberg, B. Physical Review E 2014, 89, 043013.

(78) Boţan, A.; Ulm, F.-J.; Pellenq, R. J.-M.; Coasne, B. Physical Review E 2015, 91, 032133. 


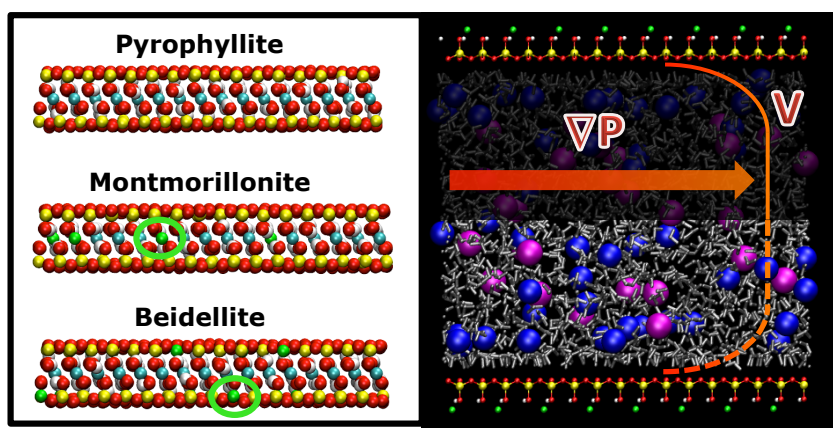

Figure 7: TOC Graphic 